\title{
Human Gut Mycobiome in IBD
}

Created by: Mario Matijasic, @Tomislav Meštrović , PHana Čipčić Paljetak, @Mihaela Perić , QAnja Barešić , QDonatella Verbanac

Version received: 16 April 2020

The human microbiota is a diverse microbial ecosystem associated with many beneficial physiological functions, as well as numerous disease etiologies. Dominated by bacteria, the microbiota also includes commensal populations of fungi, viruses, archaea, and protists. Unlike bacterial microbiota, which was extensively studied in the past two decades, these non-bacterial microorganisms, their functional roles, and their interaction with one another or with host immune system have not been as widely explored. This review covers the recent findings on the fungal communities of the human gastrointestinal microbiota, termed the "mycobiome", and their involvement in health and disease, with particular focus on the pathophysiology of inflammatory bowel disease.

\section{Introduction}

Fungi are ubiquitous in the environment and a part of all Earth's ecosystems ${ }^{[1]}$. In addition, a diverse population of commensal fungi has been recognized as a fundamental component of the human body, co-existing with other microbes within the human microbiota. [2] In contrast to the vast number of studies on the bacterial communities of the microbiota conducted in the last decades, the fungal constituents of the microbiota, the mycobiome, received much less attention. Still, recent research acknowledged human mycobiome as a dynamic community, responsive to environmental and pathophysiological changes, and playing a vital role in host metabolism as well as in maintenance of host immune homeostasis. [2][3][4][5] Human mycobiome is also implicated in various disease conditions, including inflammatory bowel disease (IBD) and its two main entities: Crohn's disease (CD) and ulcerative colitis (UC). [6][7][8][9]

\section{Human mycobiome research}

Early research of human mycobiome was based on culture-dependent techniques for the identification and characterization of commensal fungal communities. While the new molecular culture-independent next-generation sequencing (NGS) techniques proved very effective for analyzing the bacterial component of microbiota, the DNAbased sequencing studies of the human mycobiome are faced with several limitations. Fungi account for a relatively small percentage of the human microbiota, with $10^{5}$ to $10^{6}$ fungal cells per gram of fecal matter (compared to $10^{1}$ bacterial cells per gram) ${ }^{[10]}$ and only $0.1 \%$ of the 9.9 million reference genes in a current human gut microbial metagenomic reference catalog are reported to be of eukaryotic origin. [11][12] Additionally, the identification of

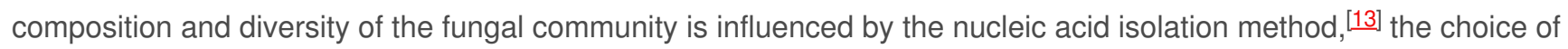
sequencing primer pairs, ${ }^{[14]}$ as well as different sequencing technologies[15][16] and bioinformatics pipelines. ${ }^{[17][18]}$ Finally, the incomplete databases for taxonomic assignment and annotation of fungal genomes present a serious difficulty in studying the human mycobiome. 14$]$

The usual molecular target for identifying fungi are the internal transcribed spacer (ITS) regions of ribosomal RNA genes. As the ITS regions are highly divergent among fungi, these regions are often sufficiently different to classify fungi to the species level. In 2012, ITS was designated as the universal DNA barcode marker for the kingdom Fungi, ${ }^{19]}$ although this approach revealed potential PCR biases.[20][21] A recent study proposed adding translational elongating factor $1 \alpha($ TEF1 $\alpha)$ as a secondary barcode to the ITS barcode in order to increase the taxonomic resolution power and enhance the accuracy of fungal species identification. [22] On the other hand, the study comparing 18S rRNA screening to ITS sequencing showed higher sensitivity of 18S rRNA RT-PCR combined with SANGER sequencing, as this method detected fungal communities in several samples which were ITS negative. [23] Currently, there is no consensus on the best methodological approach for identifying human mycobiome, and consequently the results of studies using different methods vary.

\section{Human gut mycobiome}

Human mycobiome inhabits the gastrointestinal tract but also skin, respiratory tract, genitourinary tract, as well as other mucosal surfaces in the host. The gastrointestinal tract is the most studied fungal niche in humans. Reports 


\section{Encyclopedia}

suggest that the human gut is populated by three fungal phyla, Ascomycota, Basidiomycota, and Zygomycota, 244[25] with the "core" 10 genera identified in the majority of gastrointestinal tract samples consisting of Candida (particularly C. albicans), Saccharomyces (particularly S. cerevisiae), Penicillium, Aspergillus, Cryptococcus, Malassezia (particularly M. restricta), Cladosporium, Galactomyces, Debaryomyces, and Trichosporon.[24][26] The composition of gut mycobiome seems to be dynamic over time and far more variable than the composition of bacteria, both in humans ${ }^{[27]}$ and in mice. ${ }^{[28]}$ Most studies consider fungi as commensal organisms in the gut, acquired early in life ${ }^{[29]}$ This has recently been challenged claiming that fungi do not routinely colonize the gastrointestinal tract of healthy adults, $\underline{[30]}$ instead postulating that all fungi identified in the human stool samples could be explained by their presence in the mouth or the diet. Indeed, diet is perceived as a crucial factor affecting the composition and variability of gut mycobiome. $\frac{[31]}{}$ For instance, gut mycobiome content was found to considerably differ between individuals having different dietary patterns, i.e., vegetarians and people on a conventional Western diet. [24][31] Additionally, reports suggest that the abundance of Candida in the gut positively correlated with high carbohydrate diets, and inversely correlated to consumption of total saturated fatty acids, while recent intake of short-chain fatty acids reduced the abundance of Aspergillus. ${ }^{[25]}$ Another notable finding of this study was the co-occurrence ofCandida with particular bacterial (Prevotella and Rumminococcus) and archaeal genera (Methanobrevibacter), providing support for the interkingdom syntrophic relationships in host metabolism.

One of the first indications that fungi play a role in modulating gut homeostasis is the use ofSaccharomyces boulardii as a constituent of herbal medicine traditionally utilized in Southeast Asia to reduce the severe diarrhea in patients with cholera. S. boulardii is still prescribed as a probiotic to prevent diarrhea and intestinal colonization with Clostridioides difficile following antibiotic therapy $\mathrm{y}^{[32[33]}$ and is efficient in preventing recurrent $C$. difficile infections. [34] The positive effects of $S$. boulardii come from inactivating pathogen toxins and directly inhibiting the growth and invasion of intestinal pathogens, ${ }^{[35][36]}$ as well as boosting the host immunity and exerting anti-inflammatory functions in ulcerative colitis, ${ }^{[37][38]}$ Crohn's disease, ${ }^{[37][39]}$ and $C$. difficile colitis. ${ }^{[40]} \mathrm{A}$ recent report suggests beneficial effects of another probiotic yeast, Candida kefyr, in reducing the severity of colitis in animal models by decreasing the abundance of Bacteroides and lowering IL-6 production, thus attenuating inflammation in the intestine ${ }^{[41]}$

Although fungi can exert beneficial effects to host health, the disturbance of gut mycobiota was also implicated in various gastrointestinal diseases. A recent study demonstrated no significant changes in mycobiome richness between obese and non-obese subjects; however, some specific compositional differences were noted. The most prevalent genus in non-obese individuals was Mucor, with its abundance significantly higher in non-obese individuals, and inversely correlated with metabolic markers of obesity. ${ }^{422]}$ In colorectal cancer (CRC), an alteration of fungal composition and ecology was observed, characterized by an increased Basidiomycota/Ascomycota ratio, depletion of S. cerevisiae, as well as enrichment of Rhodotorula, Malassezia, and Acremonium genera along with several Aspergillus species (including $A$. flavus, a major producer of highly toxic carcinogen aflatoxin), suggesting their possible contribution towards CRC pathogenesis. ${ }^{[43]}$ Insights into gut mycobiota playing a role in irritable bowel syndrome (IBS) were also reported. Decreased fungal diversity and dysbiosis were found in IBS patients, correlating mycobiota signature with visceral hypersensitivity, which is considered as one of the major pathophysiological features of IBS. ${ }^{[4]}$ Interestingly, treatment with fungicides could recover the visceral hypersensitivity to normal levels 4.43$]$ This finding is in accordance with a previous study that reported yeast-free diets and antifungal treatments as helpful for IBS subjects. ${ }^{[45]}$ In addition, S. boulardii was found to be effective in improving symptoms and the quality of life in IBS patients. ${ }^{46]}$

\section{Human gut mycobiome in IBD}

The majority of research on the effects of gut mycobiota in gastrointestinal diseases was however concentrated on intestinal inflammation and IBD (Table 1). Even before the advent of molecular methods and next-generation sequencing (NGS), increased levels of anti-S. cerevisiae antibodies (ASCA) were commonly found in the serum of CD patients, suggesting the host's immune responses toward intestinal fungi.[47] These antibodies, raised against mannan, a component in the fungal cell wall, were soon identified as a reliable diagnostic biomarker for $C D$ and predictors of the disease course. ${ }^{[48][49]}$ ASCA also recognize many other fungi, including Candida. [50] Indeed, reduced fungal diversity and significantly increased abundance of specific Candida species were found in pediatric IBD patients $[51]$ Sokol et al. report a similar finding in adult subjects with IBD: a decrease in gut mycobiome biodiversity and elevated Basidiomycota/Ascomycota ratio, mainly due to the increased prevalence and abundance of $C$. albicans and reduction 
of S. cerevisiae. ${ }^{[6]}$ Additional studies confirmed an increased representation of Candida species in IBD, namely $C$. tropicalis in familial $C D,[7]$ as well as $C$. glabrata in colonic biopsy samples from patients with CD![8] Besides elevated Basidiomycota/Ascomycota ratio in IBD patients in comparison to healthy controls and in IBD flares vs. IBD remission, $[6]$ fungal dysbiosis in IBD patients is also characterized by increased levels ofGibberella moniliformis, Alternaria brassicola, Aspergillus clavatus, and Cystofilobasidiaceae, ${ }^{[8]}$ while Saccharomyces cerevisiae and Malassezia sympodialis are markedly decreased. [6] Additionally, studies confirm fungal burden is increased in both CD and $U C,{ }^{[8][9]}$ with the fungal cells translocating trough the intestinal barrier during the chronic stage of colitis $9{ }^{[52]}$

Table 1. Major contributors of mycobiome changes in IBD.

\begin{tabular}{|c|c|c|}
\hline IBD type & change & reference \\
\hline$C D+U C$ & $\uparrow$ Basidiomycota/Ascomycota ratio & [6] \\
\hline$C D+U C$ & $\uparrow$ Candida albicans & [6] \\
\hline CD & $\uparrow$ Candida tropicalis & [7] \\
\hline CD & $\uparrow$ Candida glabrata & [8] \\
\hline CD & $\uparrow$ Gibberella moniliformis & [8] \\
\hline CD & $\uparrow$ Alternaria brassicola & [8] \\
\hline CD & $\uparrow$ Aspregillus clavatus & [8] \\
\hline CD & $\uparrow$ Cystofilobasidiaceae family & [8] \\
\hline$C D+U C$ & $\downarrow$ Saccharomyces cerevisiae & [6] \\
\hline$C D+U C$ & $\downarrow$ Malassezia sympodialis & [6] \\
\hline UC & $\downarrow$ fungal diversity & [6] \\
\hline$C D+U C$ & $\uparrow$ fungal burden & [8][9] \\
\hline
\end{tabular}




\section{Encyclopedia}

UC

CD $\uparrow$ fungal-bacteria interactions

$\downarrow$ fungal-bacteria interactions
[6]

[6]

Some of the studies simultaneously analyzed both the fungal and bacterial microbiota revealing that the intestinal microbial network was different in IBD patients when compared to healthy individuals. Sokol et al. identified positive correlations between the decreased abundance of $S$. cerevisiae and reduction of several bacterial genera, such as Bifidobacterium, Blautia, Roseburia, and Ruminococcus. The total number and the intensity of fungal-bacterial associations were increased in UC, with distinct interactions potentially involved in the inflammatory processes. On the other hand, weaker fungal-bacteria correlations were found in CD when compared to healthy volunteers, implying disrupted connections between two kingdoms in this disease. [6] A study by Hoarau et al. reported elevated levels of $C$. tropicalis positively correlated with Serratia marcescens and Escherichia coli in CD. Moreover, in vitro experiments confirmed these species form thicker mixed biofilm than any of the species generates individually, creating a commensal niche additionally enriched in fungal hyphae, a form of growth usually implicated in pathogenic conditions. ${ }^{[7]}$ The fact that interactions between gut bacteria and fungi are closely associated with disease was also investigated in mouse models of dextran sulfate sodium (DSS) induced colitis. Qiu et al. found that inflamed mouse intestine contained increased fungal burden in the mucosa, but decreased in the feces. The dysbiosis was characterized by elevated Wickerhamomyces, Alternaria, and Candida, together with reduced Cryptococcus, Phialemonium, and Wallemia, and unidentified Saccharomycetales genus. ${ }^{[51]}$ The study further shows mice with fungi depleted by fluconazole treatment exhibited aggravated colitis, in contrast to bacteria-depleted mice, that showed alleviated intestinal inflammation and a trend of disease remission. This finding suggests that bacteria are the major driving force in acute inflammation and fungi may act as a counterbalance in maintaining the microbial homeostasis in acute colitis. In chronic recurrent colitis however, fungi may aggravate the disease severity and translocate into locations outside the gut. ${ }^{[51]} \mathrm{A}$ recent study by Sovran et al. identified opposing effects of administratingC. albicans or S. boulardii to mice with DSS-induced colitis, resulting in increased disease severity or reduced disease symptoms, respectively. However, broad-spectrum antibiotic treatment protected the mice from colitis and $C$. albicans had no proinflammatory effect when administered to mice with disrupted bacterial microbiota, suggesting bacteria are essential for the development of colitis and $C$. albicans requires the presence of specific bacteria that trigger the intestinal inflammation to increase the disease intensity. On the other hand, mice with depleted Enterobacteriaceae exhibited normal susceptibility to colitis, but neither $C$. albicans nor $S$. boulardii could exert disease-modulating effects in this experimental setting. After reintroducing Enterobacteriaceae, both $C$. albicans and S. boulardii recovered their effects in severity of colitis. [53]

The host immune system recognizes fungi using pattern recognition receptors (PRRs), with the resulting host responses ranging from tolerance to inflammation. The key PRR for coordinating host response to fungi is Dectin-1 (CLEC7A), a C-type lectin receptor that recognizes $\beta$-glucans in the fungal cell wall. ${ }^{54]}$ Dectin-1 activates macrophages and dendritic cells, initiates phagocytosis of fungi, and induces signaling cascade via caspaseassociated recruitment domain-containing protein 9 (CARD9) and NF-kB to produce pro-inflammatory cytokines. A recent study demonstrated a central role of Dectin-1 in regulating the severity of inflammation in mouse models of DSS-induced colitis. ${ }^{[55]}$ Dectin-1 deficient mice were found to develop more severe colitis, due to the overgrowth of opportunistic fungi (i.e., Candida and Trichosporon), while treatment with antifungal drug fluconazole ameliorated the disease. [54][56] The same study revealed that a polymorphism in the Dectin-1 gene was associated with increased severity of disease in patients with UC. ${ }^{[54]}$ Recent research also identified CARD9 as the key downstream signaling molecule for the induction of immune response to fungi. $[57]$ CARD9-deficient patients are especially susceptible to fungal infections and polymorphism in CARD9 gene is associated with a higher risk of developing IBD.58] Interestingly, Candida overgrowth, which is one of the characteristic features in IBD patients, could not be positively correlated with CARD9 polymorphism. [59] Instead, Candida was hardly detectible in CARD9 deficient mice, suggesting this taxon was not the driver of dysbiosis as in dectin-1 deficient animals. ${ }^{[60]} \mathrm{IL}-17$ and IL-22 were also found to affect commensal fungal communities. A clinical study revealed secukinumab, an IL-17A antagonist, was associated with exacerbations 
in patients with CD, identifying the higher rate of fungal infections in treated subjects. 61 ] Both IL-17 and IL-22 might act as inducers of antimicrobial peptides (AMPs) in epithelial cells and were reported as protective against mucosal fungal infections. [62][63]

\section{Conclusion}

The significant role of mycobiome in maintaining human homeostasis, as well as in disease etiology, is slowly unveiling. The impact of the diverse fungal communities on human health needs to be determined in more detail in order to expand the current "bacteriocentric" view of human microbiota and provide more holistic understanding of the human superorganism. To achieve this task, two important prerequisites are essential: (1) expanding fungal reference genomes in the currently available databases for reliable identification of those microorganisms; (2) establishing uniform methods of detection for fungal commensal populations to ensure consistent and comparable evaluation of fungal abundance in different human body sites. The improved tools and the newly generated data would provide deeper insight into human mycobiome and the possibilities of its exploitation in promoting human health and ameliorating disease. Although microbiome-directed therapy is still in its infancy, studies conducted thus far suggest that direct or indirect alterations in human mycobiome may improve health outcomes in inflammatory diseases such as IBD.

\section{References}

1. Mario Matijašić; Tomislav Meštrović; Mihaela Perić; Hana Čipčić Paljetak; Marina Panek; Darija Vranešić Bender; Dina Ljubas Kelečić; Željko Krznarić; Donatella Verbanac; Modulating Composition and Metabolic Activity of the Gut Microbiota in IBD Patients. International Journal of Molecular Sciences 2016, 17, 578, 10.3390/ijms17040578.

2. Patrick C. Seed; The Human Mycobiome. Cold Spring Harbor Perspectives in Medicine 2014, 5, a019810-a019810, 10.1101/cshperspect.a019810.

3. Mathias L. Richard; Harry Sokol; The gut mycobiota: insights into analysis, environmental interactions and role in gastrointestinal diseases. Nature Reviews Gastroenterology \& Hepatology 2019, 16, 331-345, 10.1038/s41575-019-0121-2.

4. David L. Moyes; Julian R. Naglik; The mycobiome: influencing IBD severity.. Cell Host \& Microbe 2012, 11, 551-2, 10.1016/j.chom.2012.05.009.

5. Elizabeth Witherden; Saeed Shoaie; Rebecca A Hall; David L. Moyes; The Human Mucosal Mycobiome and Fungal Community Interactions. Journal of Fungi 2017, 3, 56, 10.3390/jof3040056.

6. Harry Sokol; Valentin Leducq; Hugues Aschard; Hang-Phuong Pham; Sarah Jegou; Cecilia Landman; David Cohen; Giuseppina Liguori; Anne Bourrier; Isabelle Nion-Larmurier; et al.Jacques CosnesPhilippe SeksikPhilippe LangellaDavid SkurnikMathias L RichardLaurent Beaugerie Fungal microbiota dysbiosis in IBD. Gut 2016, 66, 1039-1048, 10.1136/gutjnl-2015-310746.

7. G. Hoarau; P. K. Mukherjee; Corinne Gower; C. Hager; J. Chandra; M. A. Retuerto; Neut Christel; S. Vermeire; J. Clemente; J. F. Colombel; et al.H. FujiokaDaniel PoulainBoualem SendidM. A. Ghannoum Bacteriome and Mycobiome Interactions Underscore Microbial Dysbiosis in Familial Crohn's Disease.. mBio 2016, 7, e01250-16-16, 10.1128/mBio.01250-16.

8. Giuseppina Liguori; Bruno Lamas; Mathias L. Richard; Giovanni Brandi; Gregory Da Costa; Thomas W. Hoffmann; Massimo Pierluigi Di Simone; Carlo Calabrese; Gilberto Poggioli; Philippe Langella; et al.Massimo CampieriHarry Sokol Fungal Dysbiosis in Mucosaassociated Microbiota of Crohn's Disease Patients. Journal of Crohn's and Colitis 2015, 10, 296-305, 10.1093/ecco-jcc/jjv209.

9. Stephan J. Ott; Tanja Kühbacher; Meike Musfeldt; Philip Rosenstiel; Stephan Hellmig; Ateequr Rehman; Oliver Drews; Wilko Weichert; Kenneth N. Timmis; Kári Stefánsson; et al. Fungi and inflammatory bowel diseases: Alterations of composition and diversity. Scandinavian Journal of Gastroenterology 2008, 43, 831-841, 10.1080/00365520801935434.

10. Ron Sender; Shai Fuchs; Ron Milo; Revised Estimates for the Number of Human and Bacteria Cells in the Body. PLoS Biology 2016, 14, e1002533, 10.1371/journal.pbio.1002533.

11. Junhua Li; MetaHIT Consortium; Huijue Jia; Xianghang Cai; Huanzi Zhong; Qiang Feng; Shinichi Sunagawa; Manimozhiyan Arumugam; Jens Roat Kultima; Edi Prifti; et al.Trine Skov NielsenAgnieszka Sierakowska JunckerChaysavanh ManichanhBing ChenWenwei ZhangFlorence LevenezJuan WangXun XuLiang XiaoSuisha LiangDongya ZhangZhaoxi ZhangWeineng ChenHailong ZhaoJumana Yousuf Al-AamaSherif EdrisHuanming YangJian WangTorben Bæk HansenHenrik Bjørn NielsenSøren BrunakKarsten KristiansenFrancisco GuarnerOluf PedersenJoel DoreS Dusko EhrlichPeer BorkJun Wang An integrated catalog of reference genes in the human gut microbiome. Nature Biotechnology 2014, 32, 834-841, 10.1038/nbt.2942.

12. Junjie Qin; Ruiqiang Li; Jeroen Raes; Manimozhiyan Arumugam; Kristoffer Burgdorf; Chaysavanh Manichanh; Trine Skov Nielsen; Nicolas Pons; Florence Levenez; Takuji Yamada; et al.Daniel R MendeJunhua LiJunming XuShaochuan LiDongfang LiJianjun CaoBo WangHuiqing LiangHuisong ZhengYinlong XieJulien TapPatricia LepageMarcelo BertalanJean-Michel BattoTorben HansenD. LepaslierAllan LinnebergHenrik Bjørn NielsenEric PelletierPierre RenaultThomas Sicheritz-PonténA. Keith TurnerHongmei ZhuChang YuShengting LiMin JianYan ZhouYingrui LiXiuqing ZhangSonggang LiNan QinHuanming YangJian WangSøren BrunakJoel DoréFrancisco GuarnerKarsten KristiansenOluf PedersenJulian ParkhillJean WeissenbachMetaHIT ConsortiumPeer BorkS. Dusko EhrlichJun Wang A human gut microbial gene catalogue established by metagenomic sequencing.. Nature 2010, 464, 
59-65, 10.1038/nature08821.

13. Anna Vesty; Kristi Biswas; Michael W. Taylor; Kim Gear; Richard Douglas; Evaluating the Impact of DNA Extraction Method on the Representation of Human Oral Bacterial and Fungal Communities. PLOS ONE 2017, 12, e0169877, 10.1371/journal.pone.0169877.

14. Patricia I. Diaz; Bo-Young Hong; Amanda K. Dupuy; Linda D. Strausbaugh; Mining the oral mycobiome: Methods, components, and meaning. Virulence 2016, 8, 313-323, 10.1080/21505594.2016.1252015.

15. Daisuke Motooka; Kosuke Fujimoto; Reiko Tanaka; Takashi Yaguchi; Kazuyoshi Gotoh; Yuichi Maeda; Yoki Furuta; Takashi Kurakawa; Naohisa Goto; Teruo Yasunaga; et al.Masashi NarazakiAtsushi KumanogohToshihiro HoriiTetsuya lidaKiyoshi TakedaShota Nakamura Fungal ITS1 Deep-Sequencing Strategies to Reconstruct the Composition of a 26-Species Community and Evaluation of the Gut Mycobiota of Healthy Japanese Individuals. Frontiers in Microbiology 2017, 8, 238, 10.3389/fmicb.2017.00238.

16. Jie Tang; Iliyan D. Iliev; Jordan Brown; David M. Underhill; Vincent A. Funari; Mycobiome: Approaches to Analysis of Intestinal Fungi. Journal of Immunological Methods 2015, 421, 112-121, 10.1016/j.jim.2015.04.004.

17. Kristi Gdanetz; Gian Maria Niccolò Benucci; Natalie Vande Pol; Gregory Bonito; CONSTAX: a tool for improved taxonomic resolution of environmental fungal ITS sequences. BMC Bioinformatics 2017, 18, 538, 10.1186/s12859-017-1952-x.

18. Jonathan M. Palmer; Michelle A. Jusino; Mark T. Banik; Daniel L. Lindner; Non-biological synthetic spike-in controls and the AMPtk software pipeline improve mycobiome data. PeerJ 2018, 6, e4925, 10.7717/peerj.4925.

19. Conrad L Schoch; K.A. Seifert; Sabine Huhndorf; Vincent Robert; John L. Spouge; C. André Levesque; Wen Chen; Elena Bolchacova; Kerstin Voigt; Pedro W. Crous; et al.Andrew N. MillerMichael J. WingfieldM. Catherine AimeKwang-Deuk AnFeng-Yan BaiRobert W. BarretoMinik BegerowMarie-Josée BergeronMeredith BlackwellTeun BoekhoutMesfin BogaleNattawut BoonyuenAna R. BurgazBart BuyckL. CaiQing CaiG. CardinaliPriscila ChaverriBrian J. CoppinsAna CrespoPaloma CubasCraig CummingsUlrike DammZ. Wilhelm De BeerG. Sybren De HoogRuth Del PradoBryn DentingerJavier Diéguez-UribeondoPradeep K. DivakarBrian DouglasMargarita DueñasTuan A. DuongUrsula EberhardtJoan E. EdwardsMostafa S. ElshahedKaterina FliegerovaManohar FurtadoMiguel Ángel GarcíaZai-Wei GeGareth W. GriffithK. GriffithsJohannes Z. GroenewaldMarizeth GroenewaldM. GrubeMarieka GryzenhoutLiang-Dong GuoFerry HagenSarah HambletonRichard C. HamelinKaren HansenPaul HarroldGregory HellerCesar HerreraKazuyuki HirayamaYuuri HirookaHsiao-Man HoKerstin HoffmannValérie HofstetterFilip HögnabbaP. M. HollingsworthSeungBeom HongKentaro HosakaJos HoubrakenKaren HughesSeppo HuhtinenKevin D. HydeTimothy JamesEric M. JohnsonJoan E. JohnsonPeter R. JohnstonE.B. Gareth JonesLaura J. KellyPaul M. KirkDániel G. KnappUrmas KõljalgGábor M. KovácsCletus P. KurtzmanSara LandvikSteven D. LeavittAudra S. LiggenstofferKare LiimatainenLorenzo LombardJ. Jennifer Luangsa-ArdH. Thorsten LumbschHarinad MagantiSajeewa MaharachchikumburaMaría P. MartínTom MayAlistair R. McTaggartAndrew S. MethvenWieland MeyerJean-Marc MoncalvoSuchada MongkolsamritLászló G. NagyR. Henrik NilssonTuula Niskanenlldiko NyilasiGen Okadalzumi Okanelbai OlariagaJürgen OtteTamás PappDuckchul ParkTamás PetkovitsRaquel Pino-BodasWilliam QuaedvliegHuzefa A. RajaDirk RedeckerTara L. RintoulConstantino RuibalJullie M. Sarmiento-RamírezImke SchmittArthur SchüßlerCarol ShearerKozue SotomeFranck O.P. StefaniSoili StenroosBenjamin StielowHerbert StockingerSatinee SuetrongSung-Oui SuhG.-H. SungMotofumi SuzukiKazuaki TanakaLeho TedersooM. Teresa TelleriaEric TretterWendy A. UntereinerHector UrbinaCsaba VágvölgyiAgathe VialleThuy Duong VuGrit WaltherQi-Ming WangYan WangBevan WeirMichael WeißMerlin M. WhiteJianping XuRebecca YahrZhu L. YangA. M. YurkovJuan Carlos ZamoraNing ZhangWen-Ying ZhuangDavid Schindel Nuclear ribosomal internal transcribed spacer (ITS) region as a universal DNA barcode marker for Fungi. Proceedings of the National Academy of Sciences 2012, 109, 6241-6246, 10.1073/pnas.1117018109.

20. Eva Bellemain; Tor Carlsen; Christian Brochmann; Eric Coissac; Pierre Taberlet; H. Kauserud; ITS as an environmental DNA barcode for fungi: an in silico approach reveals potential PCR biases. BMC Microbiology 2010, 10, 189-189, 10.1186/1471-2180-10189.

21. Francesca De Filippis; Manolo Laiola; Giuseppe Blaiotta; Danilo Ercolini; Different Amplicon Targets for Sequencing-Based Studies of Fungal Diversity. Applied and Environmental Microbiology 2017, 83, e00905-17, 10.1128/aem.00905-17.

22. J. Benjamin Stielow; C. André Lévesque; Keith A. Seifert; Wieland Meyer; L. Iriny; D. Smits; R. Renfurm; G.J.M. Verkley; M. Groenewald; D. Chaduli; et al.Anne LomascoloS. WeltiL. Lesage-MeessenA. FavelA.M.S. Al-HatmiU. DammNeriman YilmazJ. HoubrakenL. LombardW. QuaedvliegM. BinderL.A.I. VaasD. VuA. M. YurkovDominik BegerowO. RoehIMarco Alexandre GuerreiroA. FonsecaKittipan SamerpitakA.D. Van DiepeningenS. DolatabadiL.F. MorenoS. CasaregolaS. MalletN. JacquesL. RosciniE. EgidiC. BizetD. Garcia-HermosoMaría P. MartínS. DengJ.Z. GroenewaldT. BoekhoutZ. Wilhelm De Beerl. BarnesT.A. DuongM.J. WingfieldG.S. De HoogP.W. CrousC.T. LewisS. HambletonT.A.A. MoussaH.S. Al-ZahraniO.A. AlmaghrabiG. Louis-SeizeR. AssabguiW. McCormickG. OmerK. DukikG. CardinaliU. EberhardtM. De VriesVincent Robert One fungus, which genes? Development and assessment of universal primers for potential secondary fungal DNA barcodes. Persoonia - Molecular Phylogeny and Evolution of Fungi 2015, 35, 242-263, 10.3767/003158515X689135.

23. K. Wagner; B. Springer; V. P. Pires; Peter M. Keller; Molecular detection of fungal pathogens in clinical specimens by $18 S$ rDNA high-throughput screening in comparison to ITS PCR and culture.. Scientific Reports 2018, 8, 6964, 10.1038/s41598-018-25129-w.

24. Heather E. Hallen-Adams; Mallory J. Suhr; Fungi in the healthy human gastrointestinal tract. Virulence 2016, 8, 352-358, 10.1080/21505594.2016.1247140.

25. Christian Hoffmann; Serena Dollive; Stephanie Grunberg; Jun Chen; Hongzhe Li; Gary D. Wu; James D. Lewis; Frederic D. Bushman; Archaea and Fungi of the Human Gut Microbiome: Correlations with Diet and Bacterial Residents. PLOS ONE 2013, 8 , e66019, 10.1371/journal.pone.0066019.

26. Mallory J. Suhr; Heather Hallen-Adams; The human gut mycobiome: pitfalls and potentials--a mycologists perspective. Mycologia 
2015, 107, 1057-1073, 10.3852/15-147.

27. Heather Hallen-Adams; Stephen Kachman; Jaehyoung Kim; Ryan M. Legge; Inés Martínez; Fungi inhabiting the healthy human gastrointestinal tract: a diverse and dynamic community. Fungal Ecology 2015, 15, 9-17, 10.1016/j.funeco.2015.01.006.

28. Serena Dollive; Ying-Yu Chen; Stephanie Grunberg; Kyle Bittinger; Christian Hoffmann; Lee Vandivier; Christopher Cuff; James D. Lewis; Gary D. Wu; Frederic D. Bushman; et al. Fungi of the Murine Gut: Episodic Variation and Proliferation during Antibiotic Treatment. PLOS ONE 2013, 8, e71806, 10.1371/journal.pone.0071806.

29. Tonya L. Ward; Dan Knights; Cheryl A. Gale; Infant fungal communities: current knowledge and research opportunities.. BMC Medicine 2017, 15, 30, 10.1186/s12916-017-0802-z.

30. Thomas Auchtung; Tatiana Y. Fofanova; Christopher J. Stewart; Andrea K. Nash; Matthew C. Wong; Jonathan Gesell; Jennifer M. Auchtung; Nadim J. Ajami; Joseph F. Petrosino; Investigating Colonization of the Healthy Adult Gastrointestinal Tract by Fungi. mSphere 2018, 3, e00092-18, 10.1128/mSphere.00092-18.

31. Lawrence A. David; Corinne F. Maurice; Rachel N. Carmody; David Gootenberg; Julie E. Button; Benjamin E. Wolfe; Alisha V. Ling; A. Sloan Devlin; Yug Varma; Michael A. Fischbach; et al.Sudha B. BiddingerRachel J. DuttonPeter J. Turnbaugh Diet rapidly and reproducibly alters the human gut microbiome. Nature 2013, 505, 559-563, 10.1038/nature12820.

32. L. V. McFarland; A randomized placebo-controlled trial of Saccharomyces boulardii in combination with standard antibiotics for Clostridium difficile disease. JAMA 1994, 271, 1913-1918, 10.1001/jama.271.24.1913.

33. Lynne V. McFarland; Systematic review and meta-analysis ofSaccharomyces boulardiiin adult patients. World Journal of Gastroenterology 2010, 16, 2202-2222, 10.3748/wjg.v16.i18.2202.

34. Sarah E. Madoff; Mariana Urquiaga; Carolyn D. Alonso; Ciarán P. Kelly; Prevention of recurrent Clostridioides difficile infection: A systematic review of randomized controlled trials. Anaerobe 2020, 61, 102098, 10.1016/j.anaerobe.2019.102098.

35. Ignazio Castagliuolo; Martin F. Riegler; Leyla Valenick; J. Thomas Lamont; Charalabos Pothoulakis; Saccharomyces boulardii Protease Inhibits the Effects of Clostridium difficile Toxins A and B in Human Colonic Mucosa. Infection and Immunity 1999, 67, 302307, 10.1128/iai.67.1.302-307.1999.

36. Reinhard Zbinden; Inhibition ofSaccharomyces boulardii(nom. inval.) on cell invasion ofSalmonella typhimuriumandYersinia enterocolitica. Microbial Ecology in Health and Disease 1999, 11, 158-162, 10.1080/089106099435736.

37. Saskia Thomas; Diana Metzke; Jürgen Schmitz; Yvonne Dörffel; Daniel C. Baumgart; Anti-inflammatory effects of Saccharomyces boulardii mediated by myeloid dendritic cells from patients with Crohn's disease and ulcerative colitis. American Journal of Physiology-Gastrointestinal and Liver Physiology 2011, 301, G1083-G1092, 10.1152/ajpgi.00217.2011.

38. Mario Guslandi; Patrizia Giollo; Pier Alberto Testoni; A pilot trial of Saccharomyces boulardii in ulcerative colitis. European Journal of Gastroenterology \& Hepatology 2003, 15, 697-698, 10.1097/00042737-200306000-00017.

39. Mario Guslandi; Gianni Mezzi; Massimo Sorghi; Pier Alberto Testoni; Saccharomyces boulardii in Maintenance Treatment of Crohn's Disease. Digestive Diseases and Sciences 2000, 45, 1462-1464, 10.1023/a:1005588911207.

40. Amir Qamar; Samer Aboudola; Michel Warny; Pierre Michetti; Charalabos Pothoulakis; J. Thomas Lamont; Ciarán P. Kelly; Saccharomyces boulardii Stimulates Intestinal Immunoglobulin A Immune Response to Clostridium difficileToxin A in Mice. Infection and Immunity 2001, 69, 2762-2765, 10.1128/iai.69.4.2762-2765.2001.

41. Kazushiro Takata; Takayuki Tomita; Tatsusada Okuno; Makoto Kinoshita; Toru Koda; Josephe A. Honorat; Masaya Takei; Kouichiro Hagihara; Tomoyuki Sugimoto; Hideki Mochizuki; et al.Saburo SakodaYuji Nakatsuji Dietary Yeasts Reduce Inflammation in Central Nerve System via Microflora. Annals of Clinical and Translational Neurology 2014, 2, 56-66, 10.1002/acn3.153.

42. M M Rodriguez; Daniel Pérez; Felipe Javier Chaves; Eduardo Esteve; Pablo Marin-Garcia; Gemma Xifra; Joan Vendrell; Mariona Jove; Reinald Pamplona; Wifredo Ricart; et al.Manuel Portero-OtinMatilde R. ChaconJosé M. Fernández-Real Obesity changes the human gut mycobiome. Scientific Reports 2015, 5, 14600, 10.1038/srep14600.

43. Olabisi Oluwabukola Coker; Geicho Nakatsu; Rudin Zhenwei Dai; William Ka Kei Wu; Sunny H Wong; Siew Chien Ng; Francis K L Chan; Joseph Jy Sung; Jun Yu; Enteric fungal microbiota dysbiosis and ecological alterations in colorectal cancer. Gut 2018, 68, 654-662, 10.1136/gutjnl-2018-317178.

44. Sara Botschuijver; Guus Roeselers; Evgeni Levin; Daisy M. Jonkers; Olaf Welting; S E M Heinsbroek; Heleen H. De Weerd; Teun Boekhout; Matteo Fornai; Ad A. Masclee; et al.Frank H.J. SchurenWouter J. De JongeJurgen SeppenRené M. Van Den Wijngaard Intestinal Fungal Dysbiosis Is Associated With Visceral Hypersensitivity in Patients With Irritable Bowel Syndrome and Rats. Gastroenterology 2017, 153, 1026-1039, 10.1053/j.gastro.2017.06.004.

45. Adele Costabile; Sara Santarelli; Sandrine P. Claus; Jeremy Sanderson; Barry N. Hudspith; Jonathan Brostoff; Jane L. Ward; Alison Lovegrove; Peter R. Shewry; Hannah E. Jones; et al.Andrew M. WhitleyGlenn R. Gibson Effect of Breadmaking Process on In Vitro Gut Microbiota Parameters in Irritable Bowel Syndrome. PLOS ONE 2014, 9, e111225, 10.1371/journal.pone.0111225.

46. Zaigham Abbas; Javed Yakoob; Wasim Jafri; Zubair Ahmad; Zahid Azam; Muhammad W. Usman; Sara Shamim; Muhammad Islam; Cytokine and clinical response to Saccharomyces boulardii therapy in diarrhea-dominant irritable bowel syndrome. European Journal of Gastroenterology \& Hepatology 2014, 26, 630-639, 10.1097/meg.0000000000000094.

47. J. Main; H. McKenzie; G. R. Yeaman; M. A. Kerr; D. Robson; C. R. Pennington; D. Parratt; Antibody to Saccharomyces cerevisiae (bakers' yeast) in Crohn's disease.. BMJ 1988, 297, 1105-1106, 10.1136/bmj.297.6656.1105.

48. J F Quinton; Boualem Sendid; D Reumaux; P Duthilleul; Antoine Cortot; B Grandbastien; G Charrier; S R Targan; J F Colombel; Daniel Poulain; et al. Anti-Saccharomyces cerevisiae mannan antibodies combined with antineutrophil cytoplasmic autoantibodies in 
inflammatory bowel disease: prevalence and diagnostic role. Gut 1998, 42, 788-791, 10.1136/gut.42.6.788.

49. E Israeli; Itamar Grotto; B Gilburd; R D Balicer; E Goldin; A Wiik; Y Shoenfeld; Anti-Saccharomyces cerevisiae and antineutrophil cytoplasmic antibodies as predictors of inflammatory bowel disease. Gut 2005, 54, 1232-1236, 10.1136/gut.2004.060228.

50. Annie Standaert; Thierry Jouault; Peggy Vandewalle; Céline Mille; Mimouna Seddik; Boualem Sendid; Jean-Maurice Mallet; JeanFrederic Colombel; Daniel Poulain; Candida albicans Is an Immunogen for Anti-Saccharomyces cerevisiae Antibody Markers of Crohn's Disease. Gastroenterology 2006, 130, 1764-1775, 10.1053/j.gastro.2006.02.009.

51. Christel Chehoud; Lindsey G. Albenberg; Colleen Judge-Golden; Christian Hoffmann; Stephanie Grunberg; Kyle Bittinger; Robert N. Baldassano; James D. Lewis; Frederic D. Bushman; Gary D. Wu; et al. Fungal Signature in the Gut Microbiota of Pediatric Patients With Inflammatory Bowel Disease. Inflammatory Bowel Diseases 2015, 21, 1948-1956, 10.1097/mib.0000000000000454.

52. Xinyun Qiu; Feng Zhang; Xi Yang; Na Wu; Weiwei Jiang; Xia Li; Xiaoxue Li; Yulan Liu; Changes in the composition of intestinal fungi and their role in mice with dextran sulfate sodium-induced colitis. Scientific Reports 2015, 5, 10416, 10.1038/srep10416.

53. Bruno Sovran; Julien Planchais; Sarah Jegou; Marjolene Straube; Bruno Lamas; Jane Mea Natividad; Allison Agus; Louise Dupraz; Jérémy Glodt; Grégory Da Costa; et al.Marie-Laure MichelPhilippe LangellaMathias L. RichardHarry Sokol Enterobacteriaceae are essential for the modulation of colitis severity by fungi.. Microbiome 2018, 6, 152, 10.1186/s40168-018-0538-9.

54. Gordon D. Brown; Dectin-1: a signalling non-TLR pattern-recognition receptor. Nature Reviews Immunology 2005, 6, 33-43, $10.1038 / n$ ri1745.

55. Iliyan D. Iliev; Vincent A. Funari; Kent D. Taylor; Quoclinh Nguyen; Christopher N. Reyes; Samuel P. Strom; Jordan Brown; Courtney A. Becker; Phillip R. Fleshner; Marla Dubinsky; et al.Jerome I. RotterHanlin L. WangDermot P. B. McGovernGordon D. BrownDavid M. Underhill Interactions Between Commensal Fungi and the C-Type Lectin Receptor Dectin-1 Influence Colitis. Science 2012, 336, 1314-1317, 10.1126/science.1221789.

56. Ce Tang; Tomonori Kamiya; Yang Liu; Motohiko Kadoki; Shigeru Kakuta; Kenshiro Oshima; Masahira Hattori; Kozue Takeshita; Takanori Kanai; Shinobu Saijo; et al.Naohito OhnoYoichiro Iwakura Inhibition of Dectin-1 Signaling Ameliorates Colitis by Inducing Lactobacillus-Mediated Regulatory T Cell Expansion in the Intestine. Cell Host \& Microbe 2015, 18, 183-197, 10.1016/j.chom.2015.07.003.

57. Anthony Plato; Sarah E. Hardison; Gordon D. Brown; Pattern recognition receptors in antifungal immunity.. Seminars in Immunopathology 2014, 37, 97-106, 10.1007/s00281-014-0462-4.

58. Bernard Khor; Agnès Gardet; Ramnik J. Xavier; Genetics and pathogenesis of inflammatory bowel disease. Nature 2011, 474, 30717, 10.1038/nature10209.

59. Harry Sokol; Kara L. Conway; Mei Zhang; Myunghwan (Mark) Choi; Bret Morin; Zhifang Cao; Eduardo J. Villablanca; Chun Li; Cisca Wijmenga; Seok Hyun Yun; et al.Hai Ning ShiRamnik J. Xavier Card9 mediates intestinal epithelial cell restitution, T-helper 17 responses, and control of bacterial infection in mice.. Gastroenterology 2013, 145, 591-601.e3, 10.1053/j.gastro.2013.05.047.

60. Bruno Lamas; Mathias L Richard; Valentin Leducq; Hang-Phuong Pham; Marie-Laure Michel; Gregory Da Costa; Chantal Bridonneau; Sarah Jegou; Thomas W Hoffmann; Jane M Natividad; et al.Loic BrotSoraya TalebAurélie Couturier-Maillardlsabelle Nion-LarmurierFatiha MerabtenePhilippe SeksikAnne BourrierJacques CosnesBernhard RyffelLaurent BeaugerieJean-Marie LaunayPhilippe LangellaRamnik J XavierHarry Sokol CARD9 impacts colitis by altering gut microbiota metabolism of tryptophan into aryl hydrocarbon receptor ligands. Nature Medicine 2016, 22, 598-605, 10.1038/nm.4102.

61. Wolfgang Hueber; Bruce E Sands; Steve Lewitzky; Marc Vandemeulebroecke; Walter Reinisch; Peter D R Higgins; Jan Wehkamp; Brian G Feagan; Michael D Yao; Marek Karczewski; et al.Jacek KarczewskiNicole PezousStephan BekGerard BruinBjoern MellgardClaudia BergerMarco LondeiArthur P BertolinoGervais TougasSimon P L TravisSecukinumab in Crohn's Disease Study Group Secukinumab, a human anti-IL-17A monoclonal antibody, for moderate to severe Crohn's disease: unexpected results of a randomised, double-blind placebo-controlled trial.. Gut 2012, 61, 1693-700, 10.1136/gutjnl-2011-301668.

62. Heather R. Conti; Fang Shen; Namrata Nayyar; Eileen Stocum; Jianing N. Sun; Matthew J. Lindemann; Allen W. Ho; Justine Hoda Hai; Jeffrey J. Yu; Ji Won Jung; et al.Scott G. FillerPatricia Masso-WelchMira EdgertonSarah L. Gaffen Th17 cells and IL-17 receptor signaling are essential for mucosal host defense against oral candidiasis. Journal of Experimental Medicine 2009, 206, 299$311,10.1084 /$ jem.20081463.

63. Melissa A. Gessner; Jessica L. Werner; Lauren M. Lilly; Michael P. Nelson; Allison E. Metz; Chad W. Dunaway; Yvonne R. Chan; Wenjun Ouyang; Gordon D. Brown; Casey T. Weaver; et al.Chad Steele Dectin-1-Dependent Interleukin-22 Contributes to Early Innate Lung Defense against Aspergillus fumigatus. Infection and Immunity 2011, 80, 410-417, 10.1128/IAI.05939-11.

\section{Keywords}

gut mycobiome; inflammatory bowel disease (IBD)

(C) 2020 by the author(s). Distribute under a Creative Commans CC BY license 